\title{
Processo de trabalho entre a Equipe de Atenção Básica e o Núcleo de Apoio à Saúde da Família
}

\author{
Work process between the Primary Care Team and the Family Health Support Centers \\ Proceso de trabajo entre el Equipo de Atención Primaria y el Núcleo de Apoyo a la Salud de la Familia
}

Isabelle Cristina Borba da Silva ${ }^{a}$, Laís Alves Bernardino da Silva ${ }^{b}$, Reíza Stéfany de Araújo e Lima ${ }^{c}$, Jailson Alberto Rodriguesc, Ana Maria Gondim Valençad, Juliana Sampaio ${ }^{\mathrm{e}}$

\section{Resumo}

O Núcleo de Apoio à Saúde da Família (NASF) atua conjuntamente às Equipes da Atenção Básica (EqAB), sendo importante compreender o processo de trabalho entre essas equipes e o planejamento das ações conjuntas. Objetivo: Analisar, na Paraíba, a organização do processo de trabalho entre o NASF e as EqAB quanto ao acesso destas às equipes NASF e ao planejamento das ações conjuntas. Métodos: Estudo descritivo de recorte transversal, com abordagem quantitativa. Os dados foram obtidos do $2^{\circ}$ Ciclo de Avaliação Externa do PMAQ-AB, realizado na Paraíba, e analisados descritivamente pelo software IBM SPSS Statistics 20.0. Resultados: $75,9 \%$ das EqAB articularam ações conjuntas com o NASF, no início das atividades de apoio; dentre essas, 85,0\% conhece o cronograma de atividades do NASF. Quanto ao acesso, o tempo de atendimento à solicitação de apoio pela EqAB é avaliado positivamente por $81,1 \%$ dos respondentes e o compartilhamento de casos ocorre em sua maioria por escrito $(70,6 \%)$, pelo agendamento direto de consultas para o profissional do NASF $(54,9 \%)$ e em discussão de casos $(54,3 \%)$. Conclusão: Há, entre as EqAB e equipe NASF, um planejamento inicial de ações conjuntas, definição de atribuições e de critérios de fluxo/encaminhamento, mas o planejamento dinâmico tende a ser menos frequente. Quanto ao acesso, há satisfação das EqAB com o apoio que recebem do NASF, mas predominam o encaminhamento por escrito e o agendamento direto como forma de compartilhamento de casos entre as equipes; a satisfação pode estar associada à divisão das demandas na $\mathrm{AB}$.

\section{Abstract}

The Support Centers for Family Health (NASF) act in conjunction with the Primary Care Teams (EqAB), and it is important to understand the work process between these teams and the planning of the joint actions. Objective: To analyze, in Paraiba, the organization of the work process between the NASF and EqAB as their access to NASF teams and the planning of joint actions. Methods: A descriptive study of cross-sectional, with a quantitative approach. The data was obtained from the 2nd External Evaluation Cycle PMAQ-AB held in Paraiba and was analyzed descriptively by IBM SPSS Statistics 20.0 Software. Results: $75.9 \%$ of EqAB articulated joint actions with NASF at the beginning of support activities, among these $85.0 \%$ NASF know the schedule of activities. As for access, the time for compliance with the EqAB support request is evaluated positively by $81.1 \%$ of respondents and case sharing occurs in writing $(70.6 \%)$, by direct appointment scheduling for the professional NASF $(54.9 \%)$ and in discussion of cases $(54.3 \%)$. Conclusion: There is, between EqAB and NASF staff, an initial planning of joint actions, defining responsibilities and flow/routing criteria, but the dynamic planning tends to be less frequent. Regarding access, there is a satisfaction of EqAB with the support they receive from NASF but what predominates is the referral in writing and direct scheduling as a way of sharing cases between the teams; satisfaction may be associated with division of the AB demands.
Como citar: Silva ICB, Silva LAB, Lima RSA, Rodrigues JA, Valença AMG, Sampaio J. Processo de trabalho entre a Equipe de Atenção Básica e o Núcleo de Apoio à Saúde da Família. Rev Bras Med Fam Comunidade. 2017;12(39):1-10. http://dx.doi.org/10.5712/rbmfc12(39)1433
Palavras-chave:

Planejamento em Saúde Estratégia Saúde da Família Atenção Primária à Saúde

Keywords:

Health Planning

Family Health Strategy

Primary Health Care
Fonte de financiamento: declaram não haver. Parecer CEP: não se aplica. Conflito de interesses: declaram não haver. Procedência e revisão por pares: revisado por pares. Recebido em: 08/12/2016. Aprovado em: 03/07/2017. 


\section{Resumen}

El Núcleo de Apoyo a la Salud de la Familia (NASF) actúa de forma conjunta con los Equipos de Atención Primaria (EqAB), y es importante entender el proceso del trabajo entre estos equipos y la planificación de acciones conjuntas. Objetivo: Analizar la organización del proceso de trabajo entre el NASF y los EqAB como su acceso a los equipos de NASF y la planificación de acciones conjuntas. Métodos: Estudio descriptivo de corte transversal, con un enfoque cuantitativo. Los datos se obtuvieron a partir de la segunda evaluación externa del PMAQ-AB celebrada en Paraíba y se analizaron descriptivamente por IBM SPSS Statistics 20.0 Software. Resultados: 75,9\% EqAB articulan acciones conjuntas con NASF al comienzo de las actividades de apoyo, entre estos 85,0\% NASF conoce el calendario de actividades. En cuanto al acceso, el tiempo para el cumplimiento de la solicitud de soporte por EqAB es evaluado positivamente por $81,1 \%$ de los encuestados y el caso se produce a través del intercambio por escrito $(70,6 \%)$, por la programación de citas directas para el profesional del NASF $(54,9 \%)$ y la discusión de los casos $(54,3 \%)$. Conclusión: Hay, entre los EqAB y el personal del NASF, una planificación inicial de acciones conjuntas, definiendo responsabilidades y flujo/criterios de enrutamiento, pero la planificación dinámica tiende a ser menos frecuente. En cuanto al acceso, hay satisfacción de los EqAB con el apoyo que reciben del NASF, pero predominan la remisión por escrito y la programación directa como una forma de compartir casos entre los equipos; la satisfacción puede estar asociada con la división de las demandas AB.

Palabras clave:

Planificación en Salud

Estrategia de Salud Familiar Atención Primaria de Salud

\section{Introdução}

Os Núcleos de Apoio à Saúde da Família (NASF), criados em 2008 pelo Ministério da Saúde (MS) por meio da Portaria no 154, são uma estratégia de fortalecimento da Atenção Básica (AB) no Brasil. Visam ampliar o poder de resolução e a abrangência das equipes de referência da Atenção Básica por meio do apoio matricial. ${ }^{1}$

Os NASF são regulamentados pela Portaria no 2.488 de $2011^{2}$ e constituem-se em equipes multiprofissionais que atuam de forma integrada com: Equipes de Atenção Básica (EqAB), Equipes de Atenção Básica para Populações Específicas e Programa Academia da Saúde. Sua atuação pressupõe uma estratégia de organização do trabalho em saúde, que acontece a partir da integração das EqAB, envolvidas na atenção às situações-problema comuns de dado território, com equipes ou profissionais com núcleos de conhecimento diferentes das equipes de referência. ${ }^{2}$

As equipes multidisciplinares assumem várias conformações, porque há uma diversidade de profissionais que podem fazer parte do escopo das equipes do NASF como: psicólogos, fisioterapeutas, assistentes sociais, profissionais da educação física, entre outros. Essa diversidade deve contribuir para a integração de saberes entre os profissionais do NASF e as equipes de referência em saúde, a partir das demandas que essa última apresentar. ${ }^{3}$

Neste sentido, o trabalho articulado entre diferentes profissionais permite não só que as ações sejam compartilhadas, mas também que se estabeleça um processo interdisciplinar, no qual progressivamente os núcleos de saberes específicos vão enriquecendo o campo comum de competências, ampliando, assim, a capacidade de cuidado de toda a equipe. ${ }^{4}$

Para atingir os objetivos a que se propõe, a Equipe NASF (EqNASF) deve interagir de maneira harmônica com a EqAB, de forma que ambas cooperem entre si para garantir uma assistência integral e resolutiva ao usuário dos serviços de saúde. Para tanto, é necessário o deslocamento de um processo de trabalho centrado em procedimentos ou em profissionais, para outro focado no usuário.

Assim, o cuidado é "o imperativo ético-político que organiza a intervenção técnico-científica", ou seja, que a integralidade do cuidado seja o centro do processo de trabalho de ambas as equipes. A existência de diversos núcleos de saberes específicos em inter-relação é um aspecto importante no 
cuidado, já que para alcançar a finalidade do trabalho em saúde é necessária a integração de saberes que deem conta dos múltiplos fatores sociais, culturais, biológicos e econômicos que interferem no processo de saúde/doença.

Neste contexto, a forma como se vivencia o processo de trabalho está intimamente ligada ao modelo assistencial executado no âmbito do cuidar em saúde. E, tal qual o nome pressupõe, o processo de trabalho é algo dinâmico, que está em constante construção, passível, a todo e qualquer momento, de ser influenciado pelos mais diversos fatores, dentre eles, o modelo vigente de produção, proteção e promoção da saúde.

Neste sentido, a análise do processo de trabalho do NASF em suas relações com as EqAB pode evidenciar potencialidades e limites do trabalho conjunto, no âmbito do fortalecimento da $A B$, e da ampliação da assistência ofertada pelas EqAB. Isto porque, por vezes, há um descompasso entre o discurso e a ação dos profissionais no trabalho em equipe. Adicionalmente, a articulação entre profissionais do NASF e EqAB tanto pode sofrer limitações na própria organização do processo de trabalho quanto pode fazer deste um potencializador da integralidade na $A B$ à saúde.

Neste contexto, a análise da organização do processo de trabalho entre os profissionais do NASF e das EqAB é relevante, porque propicia reflexão sobre fatores que são potencializadores da integralidade do cuidado, bem como aponta possíveis fragilidades do processo que reverberam em deficiências no apoio matricial ofertado. Os dados gerados pelos instrumentos do Programa Nacional de Melhoria do Acesso e da Qualidade da Atenção Básica (PMAQ-AB), aplicados às EqAB e às EqNASF, são importantes para a compreensão desse processo e da forma como ele afeta a qualidade da assistência prestada, principalmente no âmbito da $A B$.

O PMAQ-AB, criado pela Portaria no 1.654/2011 do MS, é uma estratégia de intervenção que objetiva incentivar gestores e EqAB a melhorar a qualidade do atendimento e a ampliação da oferta dos serviços de saúde na $A B .^{5,6}$ No $2^{\circ}$ Ciclo de Avaliação Externa do $P M A Q-A B$, o módulo II do instrumento de avaliação consiste em uma entrevista com os profissionais da EqAB sobre o apoio que recebem das EqNASF.

O estado da Paraíba se destaca nacionalmente por ter uma cobertura da AB estimada de 93,37\% da população, com 1.336 EqAB implantadas. Além disto, possui 273 EqNASF, das quais 143 são do tipo I, 55 do tipo II e 75 do tipo III, assumindo a quarta posição no cenário nacional em números absolutos de EqNASF. ${ }^{7}$

Neste contexto, o objetivo do presente estudo é analisar, na Paraíba, a organização do processo de trabalho entre o NASF e as EqAB no âmbito do acesso destas às EqNASF e do planejamento de suas ações conjuntas, a partir dos dados dos módulos I e II do $2^{\circ}$ Ciclo de Avaliação Externa do PMAQ-AB.

\section{Métodos}

Trata-se de um estudo descritivo de recorte transversal, com abordagem quantitativa, que tem como cenário as EqAB que recebem apoio dos NASF no estado da Paraíba. Este estado possui uma população estimada de 3.972.202 habitantes, distribuída em 223 municípios, divididos em quatro macrorregiões e 16 regiões de saúde. ${ }^{8}$ Os dados analisados no estudo foram obtidos a partir do $2^{\circ}$ Ciclo de Avaliação Externa do PMAQ-AB, realizado no estado da Paraíba por pesquisadores da Universidade Federal da Paraíba, que compõem a rede colaborativa coordenada pela UFRGS e que são autores deste estudo. 
Em 2013, quando ocorreu o 2ํㅜㄹ Ciclo de Avaliação Externa do PMAQ, havia 1.284 EqAB e 153 NASF implantados na Paraíba. ${ }^{7} \mathrm{O}$ instrumento foi aplicado a $1.149 \mathrm{EqAB}$, dentre as quais, 925 declararam receber apoio do NASF, sendo estas consideradas na análise. Os dados analisados no módulo II, relacionados à organização do processo de trabalho, foram obtidos a partir de sete questões sobre o planejamento de ações conjuntas e de seis questões sobre o acesso das EqAB aos profissionais do NASF (Quadro 1). Os dados referentes ao acesso das EqAB à Internet foram obtidos do módulo I a partir da questão ET I.11.8.

Quadro 1. Questões referentes ao planejamento de ações conjuntas e acesso das EqAB aos profissionais do NASF presentes no módulo II do 2ํㅜㄹo de Avaliação Externa do PMAQ.

\begin{tabular}{|ll|}
\hline Planejamento de ações conjuntas & Acesso das EqAB aos profissionais do NASF \\
\hline $\begin{array}{l}\text { NII 33.5 Quando os profissionais do NASF iniciaram suas } \\
\text { atividades de apoio, houve um momento para articulação das } \\
\text { ações conjuntas? }\end{array}$ & NIII 33.8 Como é feito o contato da sua equipe com o NASF? \\
NII 33.6 O que foi discutido nesse planejamento inicial? & NIII 33.9 Em média, quantos dias o NASF demora para atender \\
uma solicitação de apoio de sua equipe? & NIII 33.10 O NASF atende às solicitações da sua equipe em \\
NIII 33.11 Você conhece o cronograma de atividades do NASF & tempo adequado? \\
com sua equipe? & NIII 33.12 Todos profissionais do NASF têm garantido \\
NIII 33.14 Sua equipe e o NASF criaram critérios em relação aos \\
$\begin{array}{l}\text { atendimentos a serem realizados pelos profissionais do NASF? } \\
\text { NIII 33.16 Existem critérios e formas definidas e pactuadas } \\
\text { entre sua equipe e o NASF para acionar o apoio em situações }\end{array}$ & $\begin{array}{l}\text { NIII 33.13 Qual a periodicidade dos encontros/atividades dos } \\
\text { imprevistas? }\end{array}$ \\
$\begin{array}{ll}\text { NIII 33.16.1 Nessas situações imprevistas, sua equipe consegue } \\
\text { fazer contato com o NASF? }\end{array}$ & $\begin{array}{l}\text { NIII 33.15 Diante de um caso no qual há necessidade de apoio, } \\
\text { qual a forma mais frequente da sua equipe compartilhar o caso }\end{array}$ \\
$\begin{array}{ll}\text { NIII 33.16.2 Por quais meios os profissionais do NASF podem ser } \\
\text { acionados nessas situações? }\end{array}$ & \\
\hline NASF: Núcleo de Apoio à Saúde da Família; EqAB: Equipes da Atenção Básica; PMAQ: Programa Nacional de Melhoria do Acesso e da Qualidade da \\
Atenção Básica.
\end{tabular}

Os dados foram analisados descritivamente pelo software IBM SPSS Statistics 20.0, verificando-se as frequências absolutas e relativas de respostas às questões de interesse do estudo.

\section{Resultados}

A análise dos dados do processo de trabalho das EqAB e do NASF, no âmbito do planejamento das ações, permitiu identificar que a maioria das EqAB (75,9\%) articulou ações conjuntas com o NASF quando estes iniciaram suas atividades de apoio.

No contexto do planejamento das ações, no início das atividades, foram realizadas discussões relacionadas às pactuações das atividades a serem desenvolvidas pela EqNASF $(91,4 \%)$, às funções e atribuições inerentes às equipes $(86,2 \%)$, aos critérios e fluxos para encaminhamento de usuários às EqNASF (82,3\%), aos critérios e fluxos para encaminhamento de usuários a outros serviços/pontos de atenção $(78,1 \%)$ e objetivos, metas e resultados da atuação do NASF $(75,9 \%)$.

A maioria das EqAB entrevistadas afirmou conhecer o cronograma de atividades do NASF no âmbito do apoio ofertado (78,1\%). Dentre as que articularam ações conjuntas no início das atividades, esse percentual é de 85,0\% e entre aquelas que não tiveram essa experiência é de 51,6\%. Entre as EqAB, 71,1\% confirmaram ter criado critérios em relação aos atendimentos a serem realizados pelos profissionais do 
NASF. Esse percentual aumenta para $84,1 \%$ entre aquelas que discutiram esse aspecto no planejamento do início das atividades com o NASF e é de apenas 31,8\% entre as que não fizeram essa discussão inicial.

No âmbito do estabelecimento de critérios e formas definidas e pactuadas para acionamento do NASF em situações imprevistas, apenas $68,8 \%$ das EqAB confirmaram a existência destes. Apesar disso, $98,2 \%$ das EqAB afirmam que conseguem contatar o NASF em situações imprevistas, sendo o principal meio de comunicação o telefone pessoal dos trabalhadores $(60,4 \%)$.

Os dados demonstram que o tempo médio, em dias, para que o NASF atenda às solicitações da EqAB é variável (Figura 1), sendo que, mais frequentemente, as respostas dos NASF ocorrem em intervalos de tempo de 1,7 e 14 dias.

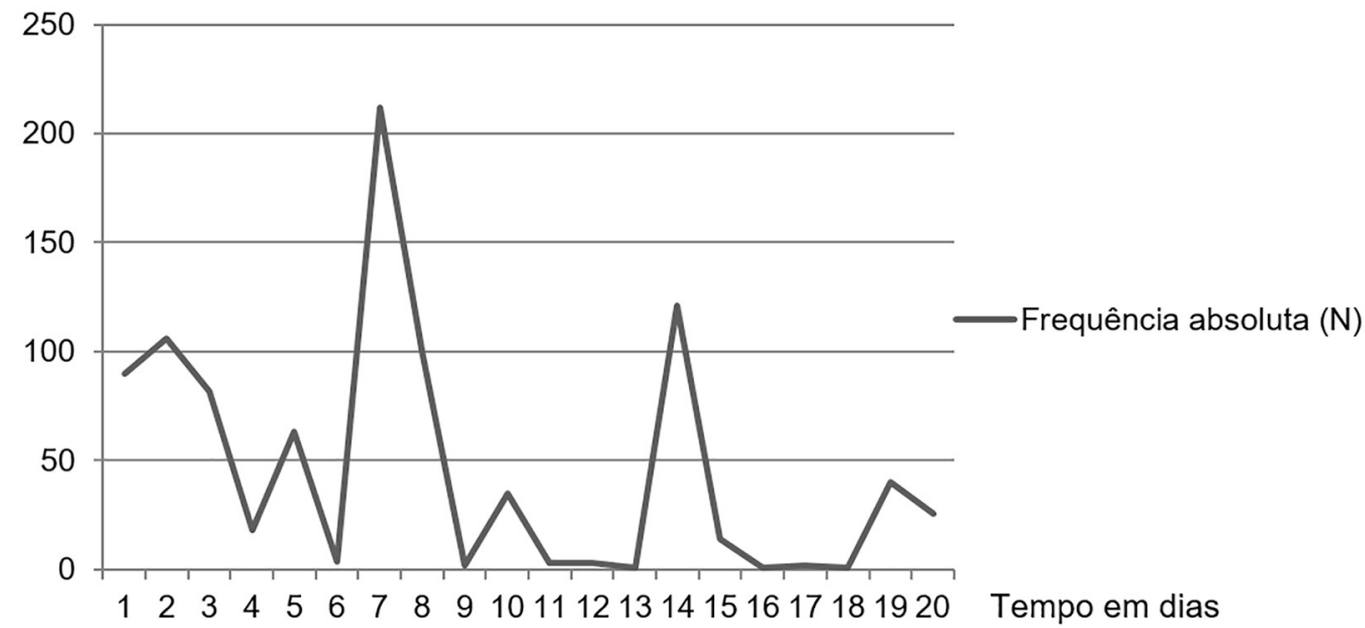

Figura 1. Espera da EqAB para o atendimento de suas demandas pelo NASF. NASF: Núcleo de Apoio à Saúde da Família; EqAB: Equipes da Atenção Básica.

Para os profissionais entrevistados, o atendimento solicitado pelas equipes de saúde ao NASF é realizado, predominantemente, sempre $(43,1 \%)$ e na maioria das vezes $(38,0 \%)$ em tempo adequado (Figura 2$)$.

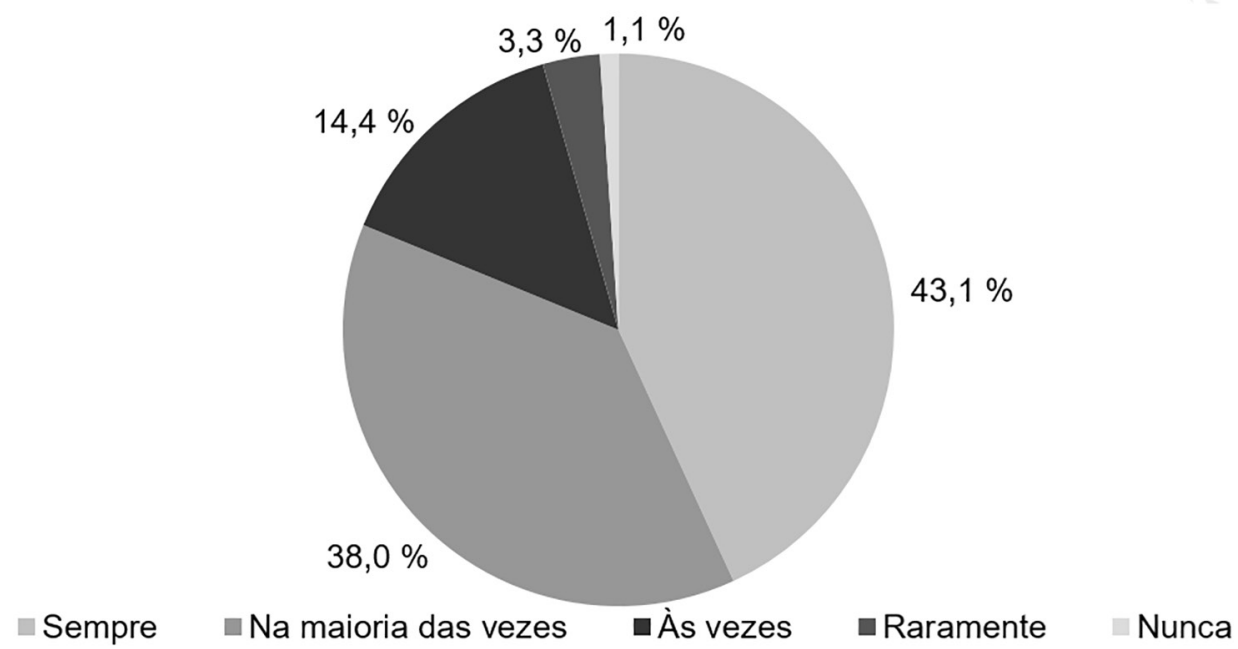

Figura 2. Avaliação da EqAB sobre o atendimento do NASF em tempo adequado. NASF: Núcleo de Apoio à Saúde da Família; EqAB: Equipes da Atenção Básica. 
A maioria dos profissionais declarou compartilhar os casos com o NASF por meio de encaminhamentos por escrito $(70,6 \%)$, pelo agendamento de consultas diretamente na agenda do profissional do NASF $(54,9 \%)$ e em discussão de casos $(54,3 \%)$ (Tabela 1$)$.

Tabela 1. Forma mais frequente de compartilhamento de casos entre a equipe e o NASF.

\begin{tabular}{lcc}
\hline Resposta & Frequência Absoluta (N) & Frequência Relativa (\%) \\
\hline Encaminhamentos por escrito & 653 & 70,6 \\
Agendamento de consultas diretamente na agenda do profissional NASF & 508 & 54,9 \\
Discussão de casos & 502 & 54,3 \\
Consultas compartilhadas & 322 & 34,8 \\
Outros & 111 & 12,00 \\
\hline
\end{tabular}

NASF: Núcleo de Apoio à Saúde da Família.

Cotidianamente, a maior parte das EqAB mantém contato com o NASF por telefone $(64,0 \%)$, em dias programados de atividades presenciais $(60,1 \%)$ e diretamente, a qualquer momento na própria UBS $(38,7 \%)$ (Tabela 2$)$.

Tabela 2. Meio de contato da EqAB com a EqNASF.

\begin{tabular}{|c|c|c|c|c|}
\hline \multirow{2}{*}{ Meio de contato da equipe com o NASF } & \multicolumn{2}{|c|}{ Sim } & \multicolumn{2}{|c|}{ Não } \\
\hline & $\mathbf{N}$ & $\%$ & $\mathbf{N}$ & $\%$ \\
\hline Por telefone & 592 & 64,0 & 333 & 36,0 \\
\hline Em dias programados de atividades presenciais & 556 & 60,1 & 369 & 39,9 \\
\hline Diretamente, a qualquer momento na própria UBS & 358 & 38,7 & 567 & 61,3 \\
\hline Mensagem de texto no celular & 169 & 18,3 & 756 & 81,7 \\
\hline Outro & 171 & 18,5 & 754 & 81,5 \\
\hline Por e-mail & 111 & 12,0 & 814 & 88,0 \\
\hline
\end{tabular}

NASF: Núcleo de Apoio à Saúde da Família; EqAB: Equipes da Atenção Básica.

No âmbito da informatização das UBS, a maioria das EqAB $(74,8 \%)$ não dispõe de acesso à internet.

\section{Discussão}

\section{Resumo dos principais achados do estudo}

No âmbito do acesso das EqAB às EqNASF, a análise dos dados permite visualizar que a maior parte das equipes obtém resposta do NASF em até uma semana e que a maioria refere satisfação com o tempo de resposta. Isso pode ser atribuído, principalmente, a dois fatores: a existência de demandas das EqAB que não requerem urgência e/ou à frequência com que os profissionais do NASF comparecem à Unidade Básica de Saúde (UBS), por exemplo, uma vez por semana ou por mês.

Os resultados demonstram ainda que algumas equipes conseguem ter acesso ao NASF, a qualquer momento, diretamente na UBS, o que sugere a organização de um processo de trabalho intra e inter equipes que busca a resolução rápida das demandas da UBS. Apesar disso, é preocupante que a maioria das EqAB compartilhem casos com o NASF por intermédio de encaminhamentos por escrito e agendamento de consultas diretamente na agenda do profissional da EqNASF, em detrimento das discussões de casos 
e consultas compartilhadas, uma vez que os dois últimos são características básicas do apoio matricial. Tal situação pode apontar para o caráter clínico-assistencial, ${ }^{9}$ predominante do NASF, bem como para a possível transferência do cuidado para esta equipe, que foge da lógica do apoio matricial. ${ }^{3}$

No âmbito do planejamento de ações conjuntas, constata-se que 75,9\% das EqAB articularam ações conjuntas com o NASF desde o momento em que este começou suas atividades na unidade, o que reflete um fator favorável à integração das equipes. Este dado é relevante, posto que, na ESF, o trabalho em equipe necessita de responsabilização compartilhada do cuidado por profissionais de diferentes saberes para gerar impacto sobre os diversos fatores que interferem no processo saúde-doença. ${ }^{10,11}$

Embora a maioria das EqAB refira ter pactuado com o NASF as atividades a serem realizadas pelo mesmo no momento de sua implantação, um percentual menor dessas equipes afirmou conhecer o cronograma atual de atividades do NASF. É preciso considerar, ainda, que mesmo essas equipes têm maior conhecimento sobre as atividades do NASF quando comparadas àquelas que não articularam as ações conjuntas no início do apoio.

Este conjunto de dados demonstra a importância do planejamento e pactuação inicial do trabalho a ser desenvolvido pelas equipes, mas evidencia também a necessidade deste processo de planejamento ser dinâmico e sistemático, de tal forma que mantenha as pactuações vivas e úteis às demandas cotidianas do processo de trabalho entre as equipes. Assim, a fragilidade na dinamicidade e efetivação do planejamento das ações desenvolvidas parece preocupante, pois é necessário que o trabalho seja compartilhado para que o NASF contribua para a consolidação da ESF e concretização da diretriz da integralidade, a partir de um apoio matricial efetivo. ${ }^{12}$

Ainda no âmbito do planejamento, houve EqAB que não criou critérios para os atendimentos a serem realizados pelos profissionais do NASF, e este quantitativo é maior entre aquelas que não planejaram inicialmente esses critérios. Isso pode apontar para uma fragilidade na compreensão das atribuições do NASF, ou refletir uma falha na cogestão do trabalho, na organização e no planejamento das atividades, principalmente, em relação aos objetivos do NASF, que incluem o atendimento compartilhado e individual em situações específicas. ${ }^{3}$ Este dado se agrava quando considerado que esses encaminhamentos são em sua maioria realizados por escrito, por meio de agendamentos, sem discussões dos casos entre as equipes.

No contexto do acesso da EqAB ao NASF, é preconizado pelo MS que devem ser estabelecidas formas de contato direto com os profissionais do NASF em casos urgentes ou imprevistos, podendo-se fazer uso de e-mail, telefones ou outras formas de acionamento do apoio por meio da equipe de referência. ${ }^{3}$ Os resultados apontam que $64,0 \%$ das EqAB mantêm contato com o NASF, majoritariamente, por telefone e $60,1 \%$ em dias programados de atividades presenciais. Esse dado relacionado à avaliação positiva que as EqAB fazem sobre o tempo de resposta do NASF demonstra que o apoio ofertado está satisfazendo as demandas da maioria das EqAB.

O uso do telefone pessoal pode, por um lado, demonstrar a comodidade do trabalhador em usar um recurso que está mais facilmente disponível, principalmente nos tempos atuais, em que a comunicação digital está disseminada e com baixo custo. Entretanto, também pode apontar uma fragilidade nas condições de trabalho das equipes, em especial no âmbito das comunicações. Inclusive porque, entre as $925 \mathrm{EqAB}$ com apoio do NASF avaliadas pelo PMAQ, apenas 233 têm acesso à rede de internet, o que pode indicar uma precarização na logística das UBS. 
A existência de encontros/atividades periódicas e regulares com os profissionais da NASF revela uma interação entre as equipes que deve proporcionar a troca de saberes entre os diversos profissionais, em consonância com o que é proposto para a atuação do núcleo. ${ }^{3}$

De maneira geral, a análise aponta para um processo de trabalho que aparentemente atende as demandas dos profissionais das EqSF. No entanto, esta satisfação por vezes parece estar mais atrelada à capacidade das EqNASF de diminuir a pressão assistencial, ofertando atendimentos especializados, com clara transferência do cuidado, do que de ofertar apoio matricial com compartilhamento do cuidado por meio de discussões de casos e desenvolvimento de ações conjuntas e articuladas.

Fortalezas e limitações do estudo

A análise de questões relacionadas ao planejamento de ações conjuntas e ao acesso das EqAB às EqNASF nos fornece um panorama do processo de trabalho entre estas equipes e aponta fragilidades e potencialidades do apoio matricial ofertado pelo NASF. Neste sentido, há aqui o enfoque pioneiro em questões relacionadas a pactuações de atividades conjuntas, tempo de espera da EqAB para ter suas demandas atendidas pela EqNASF, meio de acesso a esta em situações imprevistas, formas de compartilhamento de casos e criação e pactuação de critérios/fluxos para encaminhamentos.

Embora o $2^{\circ}$ ciclo de avaliação externa do PMAQ-AB nos forneça informações relevantes que permitem construir o perfil de composição do NASF, no estado da Paraíba, bem como a caracterização de sua atuação nos âmbitos técnico-pedagógico e clínico-assistencial e conhecer aspectos relevantes para a compreensão da forma como se organiza o processo de trabalho entre o NASF e as EqAB apoiadas, a pesquisa a partir de dados secundários possui, dentre suas limitações, a impossibilidade de contemplar a dinamicidade do processo de trabalho e de observar as relações humanas inerentes a ele.

Comparação com a literatura já existente

O NASF no âmbito do apoio matricial deve trabalhar conjuntamente com a EqAB, compartilhando os desafios enfrentados por esta e contribuindo para o aumento da resolutividade e a efetivação da coordenação integrada do cuidado na AB. Para tanto, deve priorizar o atendimento compartilhado e as intervenções específicas do profissional do NASF com os usuários após negociação com os profissionais das EqAB. ${ }^{13,14}$

Embora as diretrizes preconizem o trabalho conjunto, os dados sugerem um déficit no planejamento contínuo de ações conjuntas, bem como uma atuação individualizada dos profissionais do NASF junto à população adscrita. Isto é evidenciado pela forma de compartilhamento de casos entre as equipes.

Estes resultados corroboram com outros estudos sobre o desenvolvimento do apoio do NASF em cidades da Paraíba, que trazem inclusive acepções de apoio para além das clínico-assistencial e técnico-pedagógico, e sugerem um forte caráter assistencial no trabalho dessas equipes.9.15

Implicações para a pesquisa na área e/ou para a prática dos profissionais

A visualização da organização do processo de trabalho entre EqSF e EqNASF é relevante para os próprios profissionais das equipes, bem como para os gestores, porque permite que estes reconheçam as fragilidades que tornam a organização do processo de trabalho uma barreira à prática do cuidado integral 
e resolutivo, bem como os fatores fortalecedores da ESF e que verdadeiramente dão sentido à existência do apoio matricial.

Este estudo propicia o debate sobre a necessidade da continuidade do planejamento conjunto e do desenvolvimento das ações compartilhadas entre NASF e EqAB, que é estratégico para aumentar a resolutividade e a continuidade do cuidado. Além disso, problematiza a forma como se dá este compartilhamento, uma vez que o encaminhamento por escrito e o agendamento de consultas diretamente na agenda do profissional do NASF, que ocorrem com alta frequência no estado da Paraíba, fogem à lógica do apoio matricial.

\section{Conclusão}

A Paraíba, por possuir nacionalmente a quarta maior cobertura de EqNASF, apresenta-se como um importante cenário para estudos que busquem compreender o desenvolvimento do trabalho dos NASF junto às EqAB. Desta forma, o presente estudo torna-se relevante na medida em que possibilita a análise da percepção das EqAB sobre o apoio do NASF no que se refere ao seu acesso ao apoio ofertado e ao planejamento de suas ações conjuntas.

O trabalho em tela põe em evidência algumas potencialidades e fragilidades no planejamento e execução das ações conjuntas das equipes da AB e NASF, destacando a existência de boa comunicação entre tais equipes, mas também uma tendência expressiva para a transferência do cuidado entre as equipes, em detrimento do desenvolvimento do trabalho compartilhado, que promova a integralidade da atenção e ampliação da capacidade técnica da equipe de referência.

O estudo contribui, assim, para a compreensão do trabalho desenvolvido entre as EqAB e EqNASF, evidenciando importante configuração do modelo de apoio ofertado. Entretanto, o mesmo apresenta as limitações de um estudo a partir de dados secundários, que limita a compreensão da complexidade dos processos de trabalho desenvolvidos por essas equipes. Desse modo, acredita-se que os resultados encontrados possam subsidiar novas pesquisas e ressalta-se a necessidade da ampliação das discussões sobre o processo de trabalho das EqAB e EqNASF, não só em nível estadual, mas também nacional, a fim de compreender a efetiva relevância destes núcleos para o fortalecimento da ESF, e consequentemente, da $A B$.

\section{Referências}

1. Brasil. Ministério da Saúde. Portaria № 154, de 24 de janeiro de 2008. Cria os Núcleos de Apoio à Saúde da Família - NASF. [Internet]. Diário Oficial da República Federativa do Brasil;2008. [acesso 2016 Nov 22]. Disponível em: http://sislex.previdencia.gov.br/paginas/66/ MPS/2008/154.htm

2. Brasil. Ministério da Saúde. Portaria № 2.488, de 21 de outubro de 2011. Aprova a Política Nacional de Atenção Básica, estabelecendo a revisão de diretrizes e normas para a organização da Atenção Básica, para a Estratégia Saúde da Família (ESF) e o Programa de Agentes Comunitários de Saúde (PACS) [Internet]. Diário Oficial da República Federativa do Brasil; 2011. [acesso 2016 Nov 22]. Disponível em: http://bvsms.saude.gov.br/bvs/saudelegis/gm/2011/prt2488_21_10_2011.html

3. Brasil. Ministério da Saúde, Secretaria de Atenção à Saúde. Núcleo de Apoio à Saúde da Família. Cadernos de Atenção Básica no 39 [Internet]. Brasília: Ministério da Saúde;2014. [acesso 2016 Nov 22]. Disponível em: http://189.28.128.100/dab/docs/portaldab/publicacoes/ caderno_39.pdf

4. Brasil. Ministério da Saúde, Secretaria de Atenção à Saúde. Política Nacional de Atenção Básica. Série E. Legislação em Saúde [Internet] Brasília: Ministério da Saúde; 2012. [acesso 2016 Nov 22]. Disponível em: http://189.28.128.100/dab/docs/publicacoes/geral/pnab.pdf 
5. Brasil. Ministério da Saúde. Portaria GM № 1.654 de 19 de julho 2011. Institui no âmbito do Sistema Único de Saúde, o Programa Nacional de Melhoria do Acesso e da Qualidade da Atenção Básica (PMAQ-AB) e o Incentivo Financeiro do PMAQ-AB, denominado Componente de Qualidade do Piso de Atenção Básica Variável - PABVariável [Internet]. Diário Oficial da República Federativa do Brasil; 2011. [acesso 2016 Nov 22]. Disponível em: http://bvsms.saude.gov.br/bvs/saudelegis/gm/2011/prt1654_19_07_2011.html

6. Pinto HA, Sousa ANA, Ferla AA. O programa nacional de melhoria do acesso e da qualidade da atenção básica: várias faces de uma política inovadora. Saúde Debate [Internet].2014;38(n.spe):358-72. [acesso 2016 Nov 22]. Disponível em: http://www.scielo.br/pdf/sdeb/ v38nspe/0103-1104-sdeb-38-spe-0358.pdf. DOI: http://dx.doi.org/10.5935/0103-1104.2014S027

7. Brasil. Ministério da Saúde. Histórico de Cobertura da Saúde da Família [Internet]. 2015. [acesso 2015 Out 28]. Disponível em: http:// dab.saude.gov.br/portaldab/historico_cobertura_sf.php

8. Brasil. Instituto Brasileiro de Geografia e Estatística (IBGE). Órgãos Estaduais de Estatística, Secretarias Estaduais de Governo e Superintendência da Zona Franca de Manaus - SUFRAMA. Paraíba - PB [Internet]. 2014 Jan [acesso 2016 Nov 22]. Disponível em: http://cidades.ibge.gov.br/download/mapa_e_municipios.php?lang=\&uf=pb

9. Sampaio J, Martiniano CS, Rocha AMO, Souza Neto AA, Oliveira Sobrinho GD, Marcolino EC, et al. Núcleo de apoio à saúde da família: refletindo sobre as acepções emergentes da prática. Rev Bras Ciênc Saúde. 2013;17(1):47-54.

10. Araújo MBS, Rocha PM. Saúde da família: mudando práticas? Estudo de caso no município de Natal (RN). Ciênc Saúde Coletiva. 2009;14(Supl.1):1439-52.

11. Nascimento DDG, Oliveira MAC. Reflexões sobre as competências profissionais para o processo de trabalho nos núcleos de apoio à saúde da família. Mundo Saúde. 2010;34(1):92-6.

12. Mângia EF, Lancman S. Núcleos de Apoio à Saúde da Família: integralidade e trabalho em equipe multiprofissional. Rev Ter Ocup Univ São Paulo. 2008;19(2):i.

13. Brasil. Ministério da Saúde. Secretaria de Atenção à Saúde. Departamento de Atenção Básica. Diretrizes do NASF: Núcleo de Apoio à Saúde da Família. Série A. Normas e Manuais Técnicos/Caderno de Atenção Básica nํ27. [Internet]. Brasília: Ministério da Saúde; 2010. [acesso 2016 Nov 22]. Disponível em: http://189.28.128.100/dab/docs/publicacoes/cadernos_ab/abcad27.pdf

14. Lancman S, Gonçalves RMA, Cordone NG, Barros JO. Estudo do Trabalho e do Trabalhar no Núcleo de Apoio à Saúde da Família. Rev Saúde Pública. 2013;47(5):968-75. DOI: http://dx.doi.org/10.1590/S0034-8910.2013047004770

15. Sampaio J, Sousa CSM, Marcolino EC, Magalhães FC, Souza FF, Rocha AMO, et al. O NASF como dispositivo da gestão: limites e possibilidades. Rev Bras Ciênc Saúde. 2012;16(3):317-24.

a Universidade Federal da Paraíba (UFPB); Integrante do Programa Institucional de Bolsas de Iniciação Científica. João Pessoa, PB, Brasil. isabelleborba8@gmail.com

(Autora correspondente)

b Universidade Federal da Paraíba (UFPB). João Pessoa, PB, Brasil. laisalvesmedicina@gmail.com

c Programa de Pós-graduação em Modelos de Decisão e Saúde da Universidade Federal da Paraíba (UFPB). João Pessoa, PB, Brasil. reiza_art@ hotmail.com; jailsonalbertorodrigues@yahoo.com.br

d Universidade Federal Fluminense (UFF), Niterói, RJ, Brasil; Universidade Federal da Paraíba (UFPB)/Departamento de Clínica e Odontologia Social. João Pessoa, PB, Brasil. anamvalenca@gmail.com

e Fundação Oswaldo Cruz; Universidade Federal da Paraíba (UFPB)/Departamento de Promoção da Saúde. João Pessoa, PB, Brasil. julianasmp@ hotmail.com 